\title{
A rose on the garden fair
}

To celebrate the Rosa genomes, we invite you to imagine ways to make rosy data as well loved as the roses themselves. This is an opportunity for data modeling and new discoveries from reanalysis, as well as for data display to feed public interest in the science and culture of flowers.

W hy can't the rose genome be... more like a rose? Reading it should be full of stories, scents and colors. It should bear thorns and evoke emotions and memories. The genome sequence of this most beloved plant indeed contains thousands of years of careful, unnatural crosses and selection by human hands in China and in the Mediterranean region, culminating in combination of the two ancestral traditions in the fabulous hybrid roses we see today.

To unpick this complex weave and obtain a haploid reference genome with which to navigate the diversity of ancestral and modern roses, Mohammed Bendahmane and colleagues (https://doi.org/10.1038/ s41588-018-0110-3) essentially converted rose pollen into miniature plants and subjected these to single-DNA-molecule sequencing (described in the accompanying News \& Views by Qing Xu and ZhongJian Liu; https://doi.org/10.1038/s41588018-0141-9). Together with their main conclusions on the fundamental genotype and evolution of the Rosaceae (rose, apple, plum, raspberry) family, they examine the distribution of genes encoding the pathways that generate the characteristic floral colors and scents of various roses. We think there is plenty presented here that can be reprocessed, expanded upon, displayed and reanalyzed. For example, Supplementary Data 3 tabulates measurements of diverse levels of volatile aromatic chemicals found in the stamens and petals of roses of six genotypes, a bouquet of 61 terpenoids, phenylpropanoids, fatty acid derivatives and phenyl methyl ethers that spell 'rosy' aromas.

People have long idealized this flower as embodying ideals of loveliness and variety; as the authors of the rose genome so beautifully express it in their supplement on rose history, "These evolutionary processes have led to the emergence of traits that respond to humankind's hedonistic expectations and have represented an incredible source of diversity."

Unlike the biophilia that roses arouse, there is no corresponding 'dataphilia' for genomes that we can discern. Data can be said to be 'big', 'tidy' (J. Stat. Software 59, 1-23, 2014), 'open',
'FAIR' and even 'shared', but they are usually imagined to be useful, unlovely, tedious, pedantic and, frequently, 'dumped'.

To try to restore this balance, we would like to celebrate the beauty of rose-related datasets by providing credit and incentives to those who can connect rose data to make new discoveries, visualizations and explanations. We invite Correspondence and longer Analysis articles incorporating data models that follow the FAIR data principles (https://www.go-fair.org/fair-principles/) that make use of genomic, metabolic and phenotypic data from the published Rosa genomes and associated publicly deposited datasets together with any and all FAIR data from any other sources. We invite nongeneticists of all stripes to offer questions, use cases and make suggestions for scientific and artistic public sharing and display of the worldwide human heritage of rose culture, diversity and breeding.

Published online: 29 May 2018

https://doi.org/10.1038/s41588-018-0146-4 